\title{
Exposição a mídia e hábitos alimentares de adolescentes em uma escola privada de São Luís-MA
}

\author{
Exposure to media and eating habits of adolescents in a private school in São Luís- \\ $M A$
}

Dandara Madsen Moraes ${ }^{1}$, Iara Araujo Belfort ${ }^{2}$, Luciana Pereira Pinto Dias ${ }^{3}$, Lívia Muritiba

Pereira de Lima Coimbra ${ }^{4}$, Adrielle Zagmignan ${ }^{5}$

\begin{abstract}
Resumo: Objetivo: Este estudo teve por objetivo identificar a exposição à mídia e hábitos alimentares de adolescentes em uma escola privada de São Luís-MA. Material e Método: Estudo descritivo, feito com adolescentes de ambos os sexos, na faixa etária de 11 a 15 anos. A coleta de dados foi realizada em maio de 2017. Aplicou-se um questionário com dados sobre hábitos alimentares e tempo de exposição a mídia. Foram coletados também peso e altura. Resultados: A maioria da amostra encontrou-se eutrófica e apresentou hábitos alimentares inadequados. Foi frequente entre os adolescentes o hábito de comer na frente da televisão, celular e/ou computador. Os produtos alimentícios mais consumidos durante o tempo de exposição à mídia foram os doces $(45,0 \%)$ e fastfood $(32,5 \%)$. Conclusão: Os resultados sugerem que a exposição à mídia e os hábitos alimentares podem estar relacionados com o estado nutricional dos adolescentes.
\end{abstract}

Palavras-chave: Mídia, Estado Nutricional, Adolescente.

\begin{abstract}
Objective: This study aimed to identify and disseminate media and adolescents in a private school in São Luís-MA. Material and Method: Descriptive study, done with adolescents of both sexes, in the age group of 11 to 15 years. Data collection was performed in May 2017. A questionnaire with data on dietary habits and the exposure time of a media was applied. They were also weight and height. Results: Most of the stages were found to be eutrophic and had inadequate eating habits. It was frequent among adolescents and habit of eating in front of the television, cell phone and / or computer. The consumed foods were consumed during the time of exposure to consumption were sweet $(45.0 \%)$ and fast food $(32.5 \%)$. Conclusion: The results of exposure to the media and to the food ingredient may be related to the nutritional status of adolescents.
\end{abstract}

Key words: Media, Nutritional Status, Adolescent.

\footnotetext{
${ }^{1}$ Graduada pelo Curso de Nutrição da Universidade Ceuma.

${ }^{2}$ Graduada pelo Curso de Nutrição da Universidade Ceuma.

${ }^{3}$ Preceptora de Estágio de Nutrição Clínica - Universidade Ceuma.

${ }^{4}$ Nutricionista e Professora do Curso de Nutrição da Universidade Ceuma

${ }^{5}$ Enfermeira e Mestra em biologia parasitária pelo CEUMA. E-mail: adriellyzagmignan@hotmail.com
} 


\section{Introdução}

Estudos de acompanhamento da qualidade dos alimentos consumidos durante a adolescência são fundamentais, pois nesta fase é frequente a prática de jejum, dietas irregulares e restritas com predileção a alimentos altamente energéticos, ricos em açúcares e gorduras, em substituição a alimentos saudáveis ${ }^{1}$. E um dos principais influenciadores para que estes adolescentes adotem um padrão alimentar inadequado pode ser a mídia, através da elevada exposição de propagandas persuasivas para aquisição de gêneros industrializados ${ }^{2}$.

Dessa forma, as escolhas por alimentos de baixo valor nutritivo, têm gerado impacto negativo na saúde desses indivíduos, como: o aumento nos casos de obesidade, diabetes, hipertensão, alergias, distúrbios do sono, do crescimento, assim como deficiência no bom desenvolvimento cognitivo, que mostram serem esses problemas relevantes de saúde pública na sociedade ${ }^{3}$.

A Pesquisa Nacional de Saúde 4 assinalou informações alarmantes sobre a alimentação da população da brasileira, tais como o baixo consumo de frutas, legumes e verduras, fibras, grãos integrais. Grande parte da população não ingere os valores mínimos recomendados de sais minerais e vitaminas, além de outros nutrientes importantes para as funções vitais do organismo. Essa deficiência pode provocar diversos problemas à saúde da população.

Um local propício para o desenvolvimento destes hábitos alimentares entre os adolescentes é a escola. Observa-se que neste ambiente há um grande consumo de alimentos com elevado teor energético e pobre em nutrientes, como frituras, bebidas açucaradas e outras guloseimas, e um baixo consumo de alimentos saudáveis, como frutas e alimentos integrais, o que vai de encontro com as recomendações da alimentação escolar preconizadas no Manual de Orientação para um Lanche Saudável ${ }^{5}$.

Entre 8 e 12 anos de idade com o início da pré-adolescência, ocorre um aumento da influência da opinião dos colegas, atenção a detalhes ao se selecionar um produto, além de haver maior influência sobre as compras domésticas neste período. Dessa maneira é possível que até os 12 anos de idade, o indivíduo já tenha se familiarizado com todos os aspectos de seu perfil de consumidor ${ }^{6}$.

Neste contexto, o estudo teve pode objetivo identificar a exposição à mídia e hábitos alimentares de adolescentes em uma escola privada.

\section{Material e Métodos}

O estudo foi do tipo descritivo e a população foi constituída por adolescentes de ambos os sexos, na faixa etária de 11 a 15 anos matriculados em uma escola privada do município de São Luís - MA. Foram realizadas duas visitas para coleta de dados no mês de maio de 2017.

Os participantes foram pesados, estando descalços, de farda, em uma balança eletrônica. A estatura foi verificada com uma fita métrica, com o participante em posição ereta e pés unidos. Ambas as medidas foram coletadas por um único avaliador, sempre com os mesmos equipamentos, rotineiramente calibrados.

Para a avaliação do estado nutricional foram coletados os dados antropométricos para o cálculo do índice de massa corporal (IMC). Foram usadas as curvas de IMC para idade de 5 a 19 anos. Os percentis utilizados para classificação foram: $\geq$ Percentil 0,1 
e $<$ Percentil 3 (magreza), $\geq$ Percentil 3 e $\leq$ Percentil 85 (eutrofia), $\geq$ Percentil $85 \mathrm{e} \leq$ Percentil 97 (risco de sobrepeso), $\geq$ Percentil 97 e $\leq$ Percentil 99,9 (sobrepeso) e >Percentil 99,9 (obesidade) ${ }^{7}$.

E por fim, foi aplicado um questionário, que foi desenvolvido especialmente para estes adolescentes, a fim de avaliar se a mídia teria influência nos hábitos alimentares dos mesmos e também analisar a frequência e variedade dos alimentos consumidos, indagando-os de forma objetiva sobre a rotina de alimentação e comportamento perante as propagandas alimentícias.

A escola recebeu um Termo de Autorização para coleta dos dados. Os pais dos adolescentes receberam esclarecimentos sobre a pesquisa através de um Termo de Consentimento Livre e Esclarecido e os adolescentes um Termo de Assentimento. Ambos os termos garantiram a confidencialidade das informações adquiridas pela pesquisa aos participantes.

O estudo foi realizado na concordância com as normas que regulamentam a pesquisa em seres humanos contidas na resolução $\mathrm{n}^{\circ}$ 466/12 do Conselho Nacional de Saúde e a Declaração de Helsinque II (2000). O projeto foi submetido e aprovado pelo de Comitê de Ética em Pesquisa (CEP), sob parece de número 2.145.273

Foi realizada análise descritiva dos dados através de frequências absolutas e relativas, para as variáveis categóricas e média e desvio padrão, para as variáveis numéricas. Para verificar a associação da influência da mídia no consumo alimentar dos adolescentes foi realizado o teste Quiquadrado $\left(\chi^{2}\right)$. Nas respectivas análises estatísticas, foi adotado o nível de significância de $p<0,05$ e nível de confiança de $95 \%$. Todos os dados foram tabulados no programa EXCEL® 2010 e analisados no programa estatístico GraphPad Prism 6 software.

\section{Resultados}

A amostra foi composta por 40 escolares adolescentes, sendo 29 do sexo feminino $(72,5 \%)$ e 11 do sexo masculino $(27,5 \%)$ com idade entre 11 e 15 anos. O peso médio foi de 50,42 $\mathrm{kg} \pm 9,22$ para as meninas e $55,74 \mathrm{~kg} \pm$ 11,82 para os meninos.

Ao ser avaliado o estado nutricional dos escolares, a maioria deles encontrou-se na faixa de eutrofia $(85,0 \%)$, dentre estes $83,0 \%$ era do sexo feminino e $91,0 \%$ do sexo masculino. No entanto, para o sexo feminino $7,0 \%$ apresentaram risco de sobrepeso e $10 \%$ foram diagnosticadas com sobrepeso. Já em relação a população masculina, $9,0 \%$ dos meninos foram diagnosticados com sobrepeso. Não houve casos de obesidade (Gráfico 1).

O hábito de consumir guloseimas (A) até duas vezes na semana foi referido por $68,0 \%$ dos escolares (meninas $41,0 \%$ e meninos $2,07 \%$ ), o consumo de 3 a 4 vezes na semana foi de $53,0 \%$ (meninas $17,0 \%$ e meninos $36,0 \%)$.

O hábito da ingestão de frutas e verduras $(B)$ de 2 a 3 vezes por semana foi citado por $88,0 \%$ dos estudados (meninas $24,0 \%$ e meninos $64,0 \%$ ) e $41,0 \%$ raramente tinham esse hábito alimentar (meninas $14,0 \%$ e meninos $27,0 \%$ ).

Quanto à rotina de realizar suas refeições em frente à TV/celular/computador (C), 70,0\% dos estudantes relataram que sempre tinham esse hábito, (meninas 34,0\% e meninos 36,0\%) (Gráfico 2). 


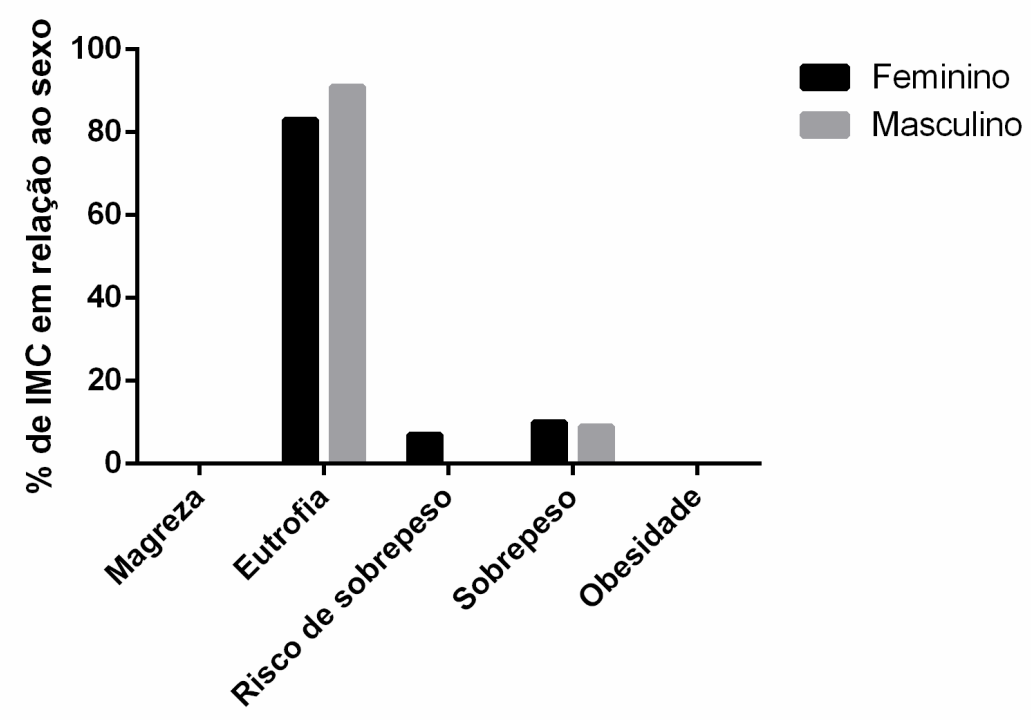

Gráfico 1. Classificação do IMC em relação ao sexo de adolescentes escolares, São Luís-MA, 2017.
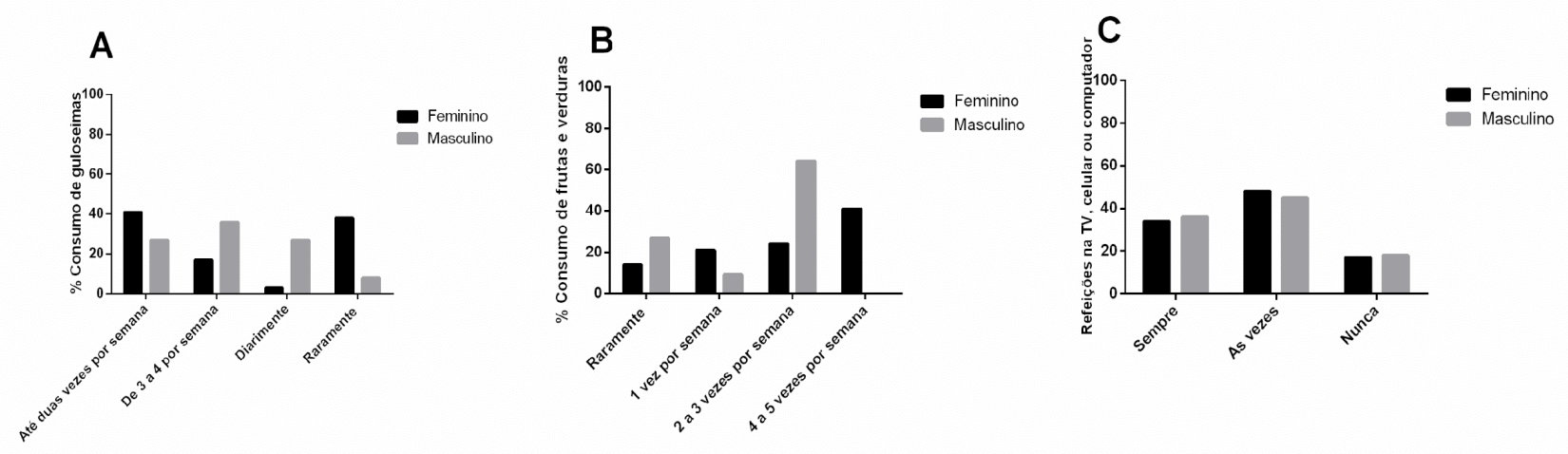

Gráfico 2. Consumo de guloseimas, frutas e verduras e hábito de realizar as refeições junto a televisão, computador e/ou celular de adolescentes escolares, São Luís-MA, 2017.
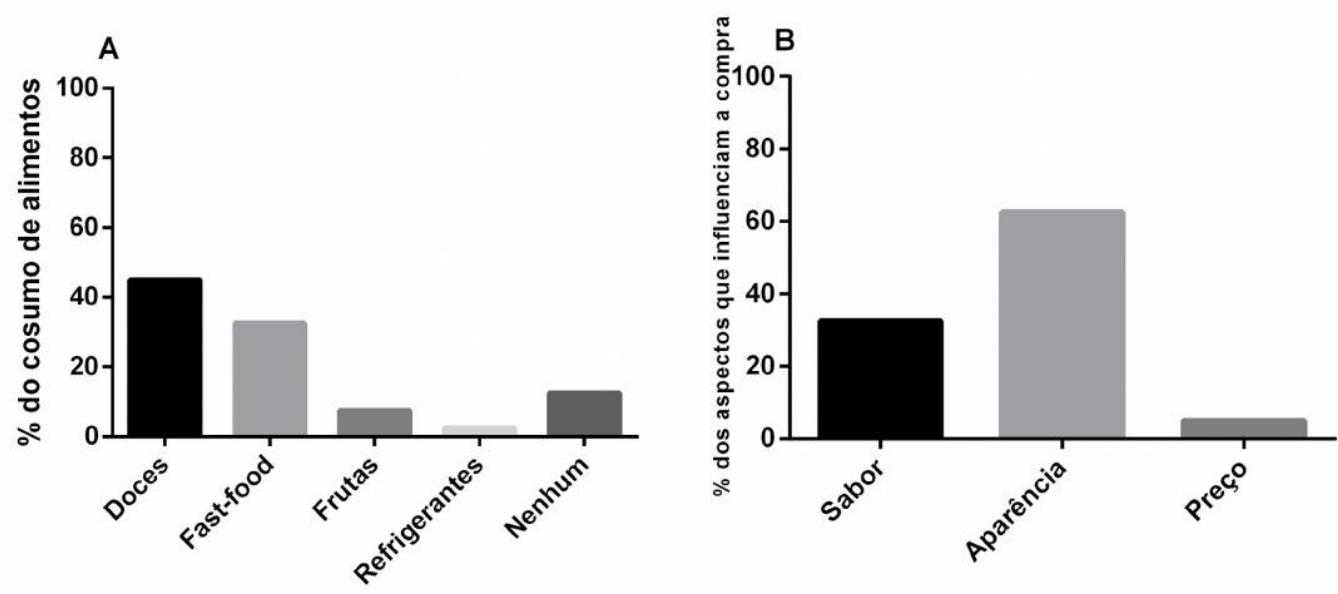

Gráfico 3. Alimentos visualizados pela mídia que costumavam comprar (A) e fatores que influenciam a compra (B) de adolescentes escolares, São Luís-MA, 2017. 
Quando questionados sobre quais os alimentos visualizados através da mídia que eles costumavam adquirir, foram mencionados doces $(45,0 \%)$, fast-foods $(32,5 \%)$, frutas $(7,5 \%)$ e refrigerantes (2,5\%) (Gráfico 3).

\section{Discussão}

No presente estudo, pôde-se constatar que quanto ao estado nutricional dos escolares, ambos os sexos encontraram-se eutróficos. Dados semelhantes foram demonstrados na pesquisa realizada por Soares e colaboradores ${ }^{7}$, onde predominantemente ambos os gêneros encontraram-se eutróficos (meninas: 67,4\%; meninos $64,5 \%$ ).

Em contraste, Porto e colaboradores $^{8}$ em seu estudo realizado com alunos de escolas privadas e públicas, verificaram que $49,0 \%$ da amostra de escolares da rede privada encontravam-se em estado de obesidade ou risco de obesidade. Nos escolares da rede pública, $51,0 \%$ da amostra foram diagnosticados com obesidade e sobrepeso. Fatores estes associados ao consumo frequente de alimentos fast-food.

Acredita-se que as boas rotinas alimentares nesta fase de transição fazem-se importantes já que contribuem para $o$ crescimento e desenvolvimento, evitando ainda a ocorrência de doenças crônicas não transmissíveis no futuro ${ }^{9}$.

O hábito de consumir guloseimas foi elevado entre os adolescentes do presente estudo. Constata-se que uma dieta com elevado consumo desses produtos ricos em açúcares e gorduras saturadas contribui para o aumento de doenças crônicas, bem como 0 excesso de peso ${ }^{10}$.

Resultados diferentes foram detectados no estudo de Junior e colaboradores ${ }^{11}$, feito entre escolares, os quais verificaram que à ingestão de frutas, foi evidenciada por pouco mais da metade dos entrevistados (53,9\%). Já Souza e colaboradores ${ }^{12}$, apontaram em seu estudo que apenas $23,73 \%$ dos adolescentes relataram consumir frutas.

Quanto à rotina das refeições em frente à TV/celular/computador, a maioria dos estudantes relatou que sempre apresentam esse hábito, resultado este parecido com a pesquisa de Albuquerque ${ }^{13}$, o qual verificou que $58,0 \%$ dos estudados informaram que se alimentavam na frente do computador.

Levy e colaboradores ${ }^{14}$, em seu estudo realizado nas 26 capitais brasileiras e no Distrito Federal, associaram 0 hábito de comer enquanto se assiste televisão com o consumo de alimentos não saudáveis e com o excesso de peso, ou seja, o hábito de se alimentar em frente à televisão ou em frente a qualquer outro meio de meio de comunicação que desvie a atenção da alimentação, faz com que esses indivíduos mal percebam o que consomem, refletindo no seu estado nutricional.

Quando questionado aos adolescentes quais os alimentos visualizados através da mídia que eles costumavam adquirir, os mais mencionados foram os doces e fastfoods. Resultados semelhantes foram apresentados por Baldissera e colaboradores ${ }^{15}$ os quais viram em seu estudo que $88,8 \%$ dos adolescentes consumiam fast-food e $18,6 \%$ doces e por Santos ${ }^{16}, \quad 0$ qual também demonstrou aumento do consumo de alimentos não saudáveis $(60,6 \%)$ e redução da alimentação saudável por adolescentes escolares.

Em referência aos aspectos que influenciaram a compra de alimentos exibidos na mídia, a aparência foi $o$ aspecto que apresentou em maior proporção no quesito de chamar mais a 
atenção dos adolescentes. Segundo Santos $^{17}, \quad 85 \%$ das propagandas alimentícias anunciavam produtos contendo açúcares, óleos e gorduras, ou seja, alimentos localizados no topo da pirâmide alimentar os quais deveriam ser consumidos em menor quantidade.

Prodanov ${ }^{18}$, verificou que quanto à compra de alimentos anunciados na televisão, 4,0\% dos estudantes responderam que pediram para os pais comprarem, e $83,0 \%$ não. Entre os motivos informados, $41,0 \%$ pensaram no preço, $41,0 \%$ não gostaram dos alimentos anunciados e 18,0\% não consideraram os alimentos como saudáveis. Dos produtos alimentícios anunciados na televisão e vistos por adolescentes de 12 a 17 anos, 89,4\% eram ricos em gordura, açúcar e sódio e pobres em conteúdo nutritivo.

Desse modo, os adolescentes podem apresentar um maior risco para desenvolver excesso de peso, obesidade e doenças crônicas, especialmente diabetes, em consequência de hábitos alimentares indevidos. Os hábitos adquiridos nessa fase, bem como o excesso de peso e a obesidade neste período, podem se perpetuar até a vida adulta ${ }^{19}$.

\section{Conclusão}

Diante dos resultados encontrados, podemos concluir que os produtos alimentícios divulgados expressivamente por propagandas como doces e fast-food foram os mais consumidos. Enquanto que a ingestão de frutas e verduras tiveram um consumo abaixo do recomendado. No entanto, a amostra estudada encontrou-se com o estado nutricional dentro da normalidade.

Sendo assim, faz-se necessário investir em campanhas de educação nutricional nas escolas a fim de promover a saúde e a prevenção de doenças crônicas não transmissíveis.

\section{Referências}

1 Silva, D. C. A. et al. Percepção de adolescentes sobre a prática de alimentação saudável. Ciência e saúde coletiva. Rio de Janeiro, v. 20, n. 11, nov. 2015.

2 Lucchini, B.G.; Enes, C.C. Influência do Comportamento Sedentário Sobre o Padrão Alimentar de Adolescentes. XIX Encontro de Iniciação Científica -Anais do IV Encontro de Iniciação em Desenvolvimento Tecnológico e Inovação, PUC Campinas, 2014.

3 Zompero, A. de F. et al. A educação alimentar e nutricional nos documentos de ensino para a educação básica. Revista Ciências \& Ideias. V. 6, n.2, p. 71-82, Jul-Dez. 2015.

4 IBGE. Instituto Brasileiro de Geografia e Estatística. Pesquisa de orçamentos familiares, 2008-2009. Análise de 0 consumo alimentar pessoal no Brasil. Rio de Janeiro. . 2010.

5 Wefort, V.R.S. et al. Lanche Saudável-Manual de Orientação. Departamento Científico de Nutrologia. Sociedade Brasileira de Pediatria. São Paulo, 2012.

6 De Moura, N. C. Influência da mídia no comportamento alimentar de crianças e adolescentes. Segurança Alimentar e Nutricional, Campinas, v. 17, n. 1, p. 113122, 2010.

7 Soares, A.P. et al. Influência da televisão nos hábitos alimentares e estado nutricional de escolares da cidade de Blumenau/S. .Brazil Journal Food Technology. Nov, 2010.

8 Porto, A.C.V. et al. Consumo de fast food em crianças. Acta Pediatr Port. v. 44, n. 6, p. 301-5, 2014.

9 Cabrera, T. F. C. et al. Análise da prevalência de sobrepeso e obesidade e do nível de atividade física em crianças e adolescentes de uma cidade do Sudoeste de São Paulo. Journal of Human Growthand Develop ment. v. 24, n. 1, p. 66-67, 2014.

10 Chaves, M.I.B. Uma investigação acerca dos hábitos alimentares na adolescência. 2014. 49 f. Monografia (Especialização) - 
Faculdade de Foz do Iguaçu. Universidade Tecnológica Federal do Paraná, 2014

11 Junior, I.S. et al. Pensar a Prática, Goiânia, v. 16, n. 2, p. 320618, abr./jun. 2013.

12 Souza, P. Influência de intervenções educativas no conhecimento sobre alimentação e nutrição de adolescentes de uma escola pública. Ciência \& Saúde Coletiva. V. 22, n. 1, p. 427-435, 2017.

13 Albuquerque, A.C.S. Estado nutricional e consumo alimentar de adolescentes em uma instituição pública no município de Cumaru - PE. 2016.59 f. Trabalho de Conclusão de Curso (Graduação em Nutrição) - Universidade Federal de Pernambuco, Vitória de Santo Antão, 2016.

14 Levy, R.B. et al.Consumo e comportamento alimentar entre adolescentes brasileiros: Pesquisa Nacional de Saúde do Escolar (PENSE), Ciência \& Saúde Coletiva, v.15, n.2, p.3085-3097, 2010.

15 Baldissera, G. et al. Práticas alimentares de crianças e adolescentes. Caderno pedagógico, Lajeado, v. 12, n. 1, p. 289300, 2015.

16 Santos, M. N. Comportamento alimentar: relação com a compulsão alimentar e os fatores de risco cardiovascular em adolescentes, 2014. 114 f. Dissertação (Mestrado em Saúde Pública). Universidade Estadual da Paraíba, 2014

17 Santos, C.C. A influência da televisão nos hábitos, costumes e comportamento alimentar. Cogitare Enfermagem. V. 17, n. 1, p. 65-71, Jan/Mar,2012.

18 Prodanov, S. A influência da publicidade nos hábitos alimentares de crianças em idade escolar. Revista Conhecimento Online, Novo Hamburgo, v. 8, n. 1, 2016.

19 Glória, A. P. A. P. O hábito de uma alimentação saudável na escola. 2014. 43 f. Trabalho de Conclusão de Curso (Especialização) - Universidade Tecnológica Federal do Paraná, Medianeira, 2014. 